\title{
Fatigue Life Prediction for Throwing Impeller of an Impeller Blower
}

\author{
Zhiping Zhai ${ }^{\mathrm{a}}$, Can Li ${ }^{\mathrm{a}}$, Hongmei Cui ${ }^{\mathrm{b}, *}$, Hongyu Liang ${ }^{\mathrm{a}}$, and Haiying Cheng ${ }^{\mathrm{a}}$ \\ ${ }^{a}$ College of Mechanical Engineering, Inner Mongolia University of Technology, Hohhot, 010051, China \\ ${ }^{b}$ College of Mechanical and Electrical Engineering, Inner Mongolia Agricultural University, Hohhot, 010018, China
}

\begin{abstract}
Impeller blowers are used to convey materials for various forage harvesters. As the main working component, the throwing impeller endures various static and dynamic loads while conveying the materials. This makes the throwing impeller prone to fatigue fracture, so it is very necessary to find a feasible model to estimate the fatigue life of the throwing impeller accurately. In order to obtain the accurate random cyclic load applied on the high-speed rotating impeller, the finite element analysis and the fluid-solid coupling method are adopted to calculate the stress distribution of the impeller under the combined action of the fluid-solid coupling flow field pressure, the centrifugal force, and the gravity. At the same time, the stress on the dangerous section of the impeller is measured by using the DH5909 wireless strain testing system and is compared with the calculated one. The contrast results show that the numerical calculation results are reliable. To accurately predict the fatigue life of the throwing impeller at the design stage, the two-parameter nominal stress model is deduced and combined with the linear cumulative damage model of Miner and the lognormal distribution model. Its two parameters of the average stress $S_{m}$ and the stress amplitude $S_{a}$ can be obtained through finite element analysis and do not have to be equivalent to a symmetrical cyclic load. Therefore, its precision of estimating the fatigue life is improved. By contrasting the rated and predicted fatigue lives of an impeller, it was found that the impeller's actual rated lives are closer to the predicted lives of the Goodman and Gerber twoparameter nominal stress model than those of the conventional $S$ - $N$ curve. In particular, they are closer to the calculation results of the Gerber-type two-parameter nominal stress model. This shows that the Gerber-type two-parameter nominal stress model is more accurate and suitable to predict the fatigue life of the throwing impeller. These achievements will play a significant role in further optimizing the impeller and improving its reliability.
\end{abstract}

Keywords: throwing impeller; fatigue life; two-parameter nominal stress model; Miner rule; log-normal distribution

(Submitted on October 15, 2018; Revised on November 12, 2018; Accepted on December 17, 2018)

(C) 2019 Totem Publisher, Inc. All rights reserved.

\section{List of Symbols}

$\begin{array}{ll}M & \text { Material constant } \\ C & \text { Material constant } \\ N & \text { Fatigue life } \\ S_{\max } & \text { Maximum alternating stress } \\ S_{m} & \text { Average stress } \\ S_{a} & \text { Stress amplitude } \\ R & \text { Stress ratio } \\ S_{-1} & \text { Fatigue limit of material under the action of symmetric cyclic load } \\ S_{b} & \text { Ultimate strength of material } \\ K_{S} & \text { Effective stress concentration factor } \\ \varepsilon_{S} & \text { Size factor } \\ \beta & \text { Surface quality coefficient } \\ \varphi_{S} & \text { Sensitivity coefficient of material to the asymmetry of cycle stress } \\ n & \text { Actual cyclic number } \\ D & \text { Damage fraction }\end{array}$

* Corresponding author.

E-mail address: chm123m@126.com 


$\begin{array}{ll}k & \text { Stress levels number } \\ n_{i} & \text { Actual cyclic number there are } k \text { different stress levels in one cycle } \\ S_{a i} & \text { Average stress at a constant amplitude stress level } \\ S_{m i} & \text { Stress amplitude at a constant amplitude stress level } \\ N_{i} & \text { Average number of cycles to failure at a constant amplitude stress level }\left(S_{a i}, S_{m i}\right) \\ x & \text { Random variable of fatigue life } \\ \mu & \text { Logarithmic mean deviation } \\ \sigma & \text { Logarithmic standard deviation } \\ E(X) & \text { Mean deviation } \\ \sqrt{D(X)} & \text { Standard deviation } \\ \Phi\left(\frac{\ln x-\mu}{\sigma}\right) & \text { Standard normal distribution function }\end{array}$

\section{Introduction}

An impeller blower generally consists of the throwing impeller, shell, and discharge tube. This device is widely used to convey materials in various forage harvesters because of its simplicity, reliability, easy maintenance, high capacity, and low manufacturing cost [1]. As the core working component, the throwing impeller often endures the combined action of the centrifugal force, the gravity, and the varying pressure of high-speed air flow and materials inside the impeller blower. This will accelerate the throwing impeller's fatigue, leading to fracture [2]. In recent years, most research on impeller blowers mainly focused on reducing the power consumption as well as increasing the throwing distance and efficiency [3-6]. However, relatively few data exist on the topic of investigating the fatigue life of the throwing impeller. Thus, it is very necessary to find a feasible model to accurately estimate the fatigue life of throwing impellers.

Research works on the fatigue life for the similar rotating machine are presented as follows. Dileep [7] estimated the low-cycle fatigue life of a centrifugal impeller under the engine operating conditions by using four different multi-axial fatigue damage models. The predicted lives were then compared with the experimental value obtained from the cyclic spin test. The critical plane-based models showed better prediction accuracy and capability in comparison to the maximum von Mises strain range model. Shanmugam [8] estimated the fatigue life of a titanium alloy centrifugal impeller by using the equivalent strain method and Smith Watson Topper (SWT) method and assessed the fatigue life via the cyclic spin test. The probabilistic analysis of fatigue life was carried out by using the Weibull distribution method. It was found that the equivalent strain method is nonconservative due to the exclusion of the mean stress effect. The average fatigue life of the impeller was estimated at 12000 cycles by using the SWT method, which was closer to the test results. Xu [9] analyzed the fatigue life of a remanufactured centrifugal compressor impellers by using the $S-N$ curve data and the rain-flow counting method based on FEA. It was found that the simulation results were useful for analyzing fatigue life impact factors and fatigue fracture areas, although it was incapable of providing high-precision prediction. Liao and Huang [10] investigated the fatigue life of a turbine disk by constructing Goodman and Gerber types of a generalized $\sigma-\mathrm{N}$ curved surface equation based on stress amplitude and mean stress. An aircraft engine turbine disk was presented to validate the proposed method. The results showed that the prediction of the Goodman equivalent life curve was much more accurate than that of the Gerber type model. This method also showed better accuracy for estimating the fatigue life than the conventional $S$ - $N$ curve. Pavlou [11] presented a new theory for macroscopic fatigue damage estimation based on the $S$ - $N$ curve, which was a generalization of most of the existing fatigue damage models. Saggar [12] presented a fatigue damage cumulative model under the variable load devoted for defective materials. This model was connected cycle by cycle with the $S$ - $N$ curve, and it also took into account the loading history. The predicted results of this model were compared with the experimental results for a C35 low alloy steel containing defects and those computed by Miner's model. It was demonstrated that the proposed model predicted the fatigue damage much better than the Miner rule.

From the research above, in order to ensure safe and reliable operation of the throwing impeller, the two-parameter (namely the average stress $S_{m}$ and the stress amplitude $S_{a}$ ) nominal stress model, the cumulative damage model of Miner, and the logarithm normal distribution model are combined to estimate the fatigue life of the impeller in this paper. In addition, the fluid-solid coupling method in the finite element analysis is used to accurately obtain the random cyclic dynamic stress distribution of the throwing impeller under the combined effect of the flow field pressure, centrifugal force, and gravity. The stress on the dangerous cross section of the impeller at the empty load is also tested by using the DH5909 wireless strain test system. Then, the measured stresses are compared with the simulated ones via the finite element numerical calculation. After verifying the correctness of the numerical model, the fatigue life of a throwing impeller under the working load will be predicted by calculating the variation of stress along with time at the maximum stress point of the impeller's critical section. 
The rest of this article is organized as follows. Section 2 proposes the fatigue reliability theory model for structures under the random cyclic load. Section 3 provides a numerical calculation method on the stress distribution of a high-speed rotating impeller. The fatigue life prediction of an impeller blower is presented in Section 4. Finally, Section 5 presents three conclusions.

\section{Theory of Fatigue Reliability}

\subsection{Two-Parameter Nominal Stress Model}

According to the $S$ - $N$ curve and the constant life curve of material, the two-parameter nominal stress equation including the average stress $S_{m}$, the stress amplitude $S_{a}$, and the fatigue life $N$ can be deduced as follows [12-13].

Considering that the throwing impeller belongs to the high cycle fatigue, the relationship between its maximum alternating stress $S_{\max }$ and the fatigue life of $N$ is given by

$$
S_{\max }^{m} N=C
$$

Where $m$ and $C$ are the material constants.

The relationship including the maximum alternating stress $S_{\max }$, the average stress $S_{m}$, and the stress amplitude $S_{a}$ can be expressed as follows:

$$
\begin{gathered}
S_{\max }=\frac{2 S_{m}}{1+r} \\
S_{a}=\left(\frac{1-r}{1+r}\right) S_{m}
\end{gathered}
$$

Where $r$ is the stress ratio.

Substituting Equation (2) into Equation (1), Equation (4) will be obtained as follows:

$$
S_{m}=\frac{1+r}{2}\left(\frac{C}{N}\right)^{1 / m}
$$

Substituting Equation (4) into Equation (3), $S_{a}$ can be expressed as follows:

$$
S_{a}=\frac{1-r}{2}\left(\frac{C}{N}\right)^{1 / m}
$$

Because the impeller is running at the random cyclic load, the $S-N$ curve that is more suitable for the condition of the symmetrical cyclic load needs to be revised. Thus, the Goodman formula is selected as the constant life curve, as follows:

$$
\frac{S_{a}}{S_{-1}}+\frac{S_{m}}{S_{b}}=1
$$

Where $S_{b}$ is the ultimate strength of the material and $S_{-1}$ is the fatigue limit of the material under the action of the symmetric cyclic load.

Substituting Equations (4) and (5) into Equation (6), Equation (7) can be obtained as follows:

$$
S_{-1}=\frac{S_{b}(1-r)(C / N)^{1 / m}}{2 S_{b}-(1+r)(C / N)^{1 / m}}
$$


Substituting Equation (7) into Equation (6), the average stress $S_{m}$ will be obtained as follows:

$$
S_{m}=\frac{\left[(1+r) S_{a}+(1-r) S_{b}\right](C / N)^{1 / m}-2 S_{a} S_{b}}{(1-r)(C / N)^{1 / m}}
$$

Then, the two-parameter nominal stress equation of Goodman type is expressed as follows:

$$
\left[\frac{2 S_{a} S_{b}}{(1+r) S_{a}+(1-r)\left(S_{b}-S_{m}\right)}\right]^{m} N=C
$$

If we choose the Gerber formula as the constant life curve [14],

$$
\left(\frac{S_{m}}{S_{b}}\right)^{2}+\left(\frac{S_{a}}{S_{-1}}\right)=1
$$

The two-parameter nominal stress equation of Gerber type can be similarly obtained as follows:

$$
\left\{\frac{\left[(1-r)^{2}\left(S_{b}^{2}-S_{m}^{2}\right)^{2}+4 S_{a}^{2}(1+r)^{2} S_{b}^{2}\right]^{1 / 2}-(1-r)\left(S_{b}^{2}-S_{m}^{2}\right)}{S_{a}(1+r)^{2}}\right\}^{m} N=C
$$

\subsection{Two-Parameter Nominal Stress Model of a Throwing Impeller}

If we apply the $S_{a}-S_{m}-N$ two-parameter nominal stress models of material into estimating the fatigue life of the actual throwing impellers, the factors including the stress concentration, size, surface quality, and sensitivity of impeller material to the asymmetrical cycle stress that affect the fatigue strength of the impeller must be considered [15]. The revised Goodman-type two-parameter nominal stress equation is listed as follows:

$$
\left[\frac{\left(2 \frac{K_{S}}{\varepsilon_{S} \beta}\right) S_{a} S_{b}}{(1+r)\left(\frac{K_{S}}{\varepsilon_{S} \beta}\right) S_{a}+(1-r)\left(S_{b}-\varphi_{S} S_{m}\right)}\right]^{m} N=C
$$

The revised Gerber-type two-parameter nominal stress equation is listed as follows:

$$
\left\{\frac{\left[(1-r)^{2}\left(S_{b}^{2}-\varphi_{S}^{2} S_{m}^{2}\right)^{2}+4\left(\frac{K_{S}}{\varepsilon_{S} \beta}\right)^{2} S_{a}^{2}(1+r)^{2} S_{b}^{2}\right]^{1 / 2}-(1-r)\left(S_{b}^{2}-\varphi_{S}^{2} S_{m}^{2}\right)}{\left(\frac{K_{S}}{\varepsilon_{S} \beta}\right) S_{a}(1+r)^{2}}\right\}^{m} N=C
$$

Where $K_{S}$ is the effective stress concentration factor of the impeller, $\varepsilon_{S}$ is the size factor, $\beta$ is the surface quality coefficient, and $\varphi_{S}$ is the sensitivity coefficient of the impeller material to the asymmetry of the cycle stress.

\subsection{Miner Cumulative Fatigue Damage Model}

The throwing impeller always works under the condition of the alternating loads with different frequencies and different amplitudes. Its fatigue damage will accumulate to a certain extent, leading to the impeller's fracture. Thus, the average 
stress and stress amplitude should be considered for the fatigue strength analysis. According to the two-parameter nominal stress Formula (12) and Formula (13), the fatigue damage of the impeller under the cyclic load at certain stress level $\left(S_{a}, S_{m}\right)$ can be directly calculated.

Miner's cumulative damage theory assumes that the damage is accumulated linearly. Each cycle will cause some damage to the material when the material is subjected to the higher stress than the fatigue limit. For some components under the actions of constant amplitude stress level $\left(S_{a}, S_{m}\right)$, the fatigue damage caused by each cycle is $1 / N\left(S_{a}, S_{m}\right)$. The fatigue damage caused by $n$ cycles is calculated as follows:

$$
D=\frac{n\left(S_{a}, S_{m}\right)}{N\left(S_{a}, S_{m}\right)}
$$

Where $n$ is the actual cyclic number and $N$ is the number of cycles to failure at a particular stress level $\left(S_{a}, S_{m}\right)$. Failure is expected to occur when $n$ is equal to $N$ or $D$ is equal to one.

As far as the throwing impeller at different amplitude stress levels is concerned, its damage fraction $D$ is expressed as follows under the condition that there are $k$ different stress levels applied on the impeller in one cycle and the average number of cycles to failure after $n_{i}$ cycles at a constant amplitude stress level of $S_{a i}$ and $S_{m i}$ is $N_{i}$. The fatigue damage caused by $n_{i}$ cycles is calculated as follows:

$$
D=\sum_{i=1}^{k} D_{i}=\sum_{i=1}^{k} \frac{n_{i}\left(S_{a i}, S_{m i}\right)}{N_{i}\left(S_{a i}, S_{m i}\right)}
$$

When these damage add up to one, the impeller will experience fatigue failure.

In this way, there is only one-to-one transformation between each fatigue damage and each load cycle represented by the two-parameter amplitude and mean value. Because the equivalent transformation of stresses does not need to be carried out, the estimating accuracy of the fatigue life is improved.

\subsection{Fatigue Life Lognormal Distribution Model}

Due to the randomness of the basic variables such as load, material parameters, and geometrical parameters of the impeller in the working process, the fatigue life shows discreteness. The throwing impeller's material is Q235, and its fatigue life distribution obeys the lognormal distribution according to the existing research [16].

Under the action of the cyclic load, the distribution law of the impeller's fatigue life can be expressed by the lognormal probability density function [17] as follows:

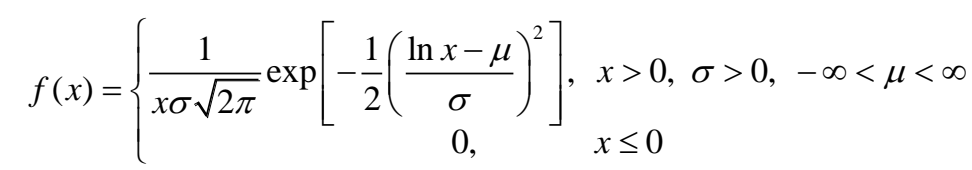

Where $x$ is a random variable of the fatigue life and $\mu$ and $\sigma$ are the logarithmic mean and standard deviation respectively.

The distribution function of the lognormal distribution is given by the following formula:

$$
F(x)=P\{X \leq x\}=\int_{0}^{x} \frac{1}{x \sigma \sqrt{2 \pi}} \exp \left[-\frac{1}{2}\left(\frac{\ln x-\mu}{\sigma}\right)^{2}\right] \mathrm{d} x
$$

Mean $E(X)$ and the standard deviation $\sqrt{D(X)}$ are calculated by using the following two formulas: 


$$
\begin{gathered}
E(X)=\exp \left(\mu+\frac{\sigma^{2}}{2}\right) \\
D(X)=\exp \left(2 \mu+\sigma^{2}\right)\left(\exp \sigma^{2}-1\right)
\end{gathered}
$$

The reliability function of the lognormal distribution is expressed as follows:

$$
P(x)=1-F(x)=\int_{x}^{\infty} \frac{1}{x \sigma \sqrt{2 \pi}} \exp \left[-\frac{1}{2}\left(\frac{\ln x-\mu}{\sigma}\right)^{2}\right] \mathrm{d} x=1-\Phi\left(\frac{\ln x-\mu}{\sigma}\right)
$$

Where $\Phi\left(\frac{\ln x-\mu}{\sigma}\right)$ is the standard normal distribution function.

\section{Fluid-Structure Coupling and Stress Analysis of the Impeller}

The flow field pressure distribution inside the impeller blower is very complex and difficult to be measured directly. In order to accurately get the flow field and load distribution on impellers, the numerical calculation of the gas-solid two-phase flow in the impeller blower is carried out by using the FLUENT. The ANSYS Workbench is also used to carry out the fluidsolid-solid (air-material-impeller) coupling process. The pressure generated by the gas-solid flow field as well as the centrifugal force and gravity are loaded on the impeller. The stress distribution of the impeller is obtained. Then, the stress of the impeller under the unloaded condition is measured to verify the numerical calculation results by using the DH5909 wireless strain test system. After that, the stress distribution of the throwing impeller under the loading condition is calculated by using the ANSYS Workbench.

\subsection{Finite Element Model and Meshing of the Impeller}

The three-dimensional modelling software Pro/E is used to build a three-dimensional solid model of the impeller. The threedimensional solid model of the impeller is then imported into HyperMesh for meshing. The numbers of cells and nodes of the entire impeller model in the finite element mesh are 26,800 and 40,532 respectively [18].

\subsection{Fluid-Structure Coupling Analysis}

The flow channel model of the impeller blower is established in Fluent at first. The large eddy simulation (LED) model is used to simulate the unsteady flow field in the impeller blower. The variation law of the instantaneous fluctuation pressure in the single-phase airflow field and the flow field pressure distribution on the impeller surface will be obtained.

Then, the Fluent and Workbench coupling analysis platform is used to exert the simulated flow field pressure as well as the centrifugal force and gravity to the coupling surface of the impeller. Constraints are also imposed on the impeller to restrict its reciprocating motions in the $X, Y$, and $Z$ directions and rotational motions around the $X$ and $Z$ axes. There is only one degree of freedom of the $Y$-axis rotation left. The impeller's speed is $1500 \mathrm{r} / \mathrm{min}$.

\subsection{Stress Analysis of the Throwing Impeller}

Under the combined action of the above loads, the equivalent stress of the impeller under the unloaded state is calculated by using the finite element method as shown in Figure 1. By using the post-processing tools in the Workbench software, the maximum stress is detected, which is $25.435 \mathrm{MPa}$ at the junction of the blade root and the stiffener. This is due to the stress concentration caused by the sharp change of the geometric shape and the thickness at the joint.

Set the stress monitoring point in the area with the biggest stress as shown in Figure 2. The stress simulation results in 0.25 seconds at the monitoring point are illustrated in Figure 3 when the impeller rotates at a speed of $1500 \mathrm{r} / \mathrm{min}$ in the unloaded and stable condition.

It can be seen from Figure 3 that the dynamic loads at the monitoring point are changing according to the random cyclic and quasi-periodic variables, and the period of the stress variation is the same as the impeller rotation period. The maximum 
stress is $8.763 \mathrm{MPa}$, the minimum is $4.721 \mathrm{MPa}$, and the average stress is $6.880 \mathrm{MPa}$.

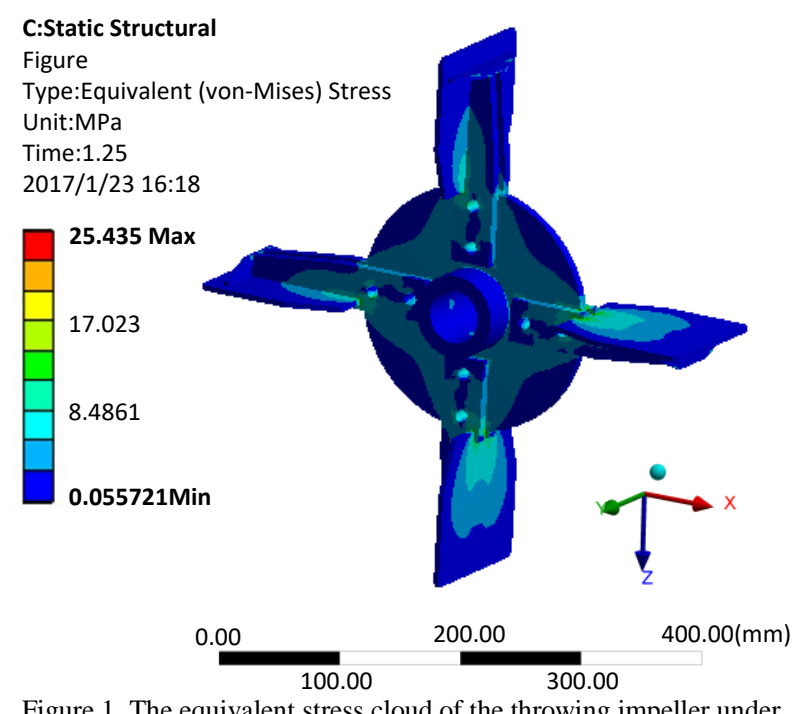

Figure 1. The equivalent stress cloud of the throwing impeller under the unloaded condition

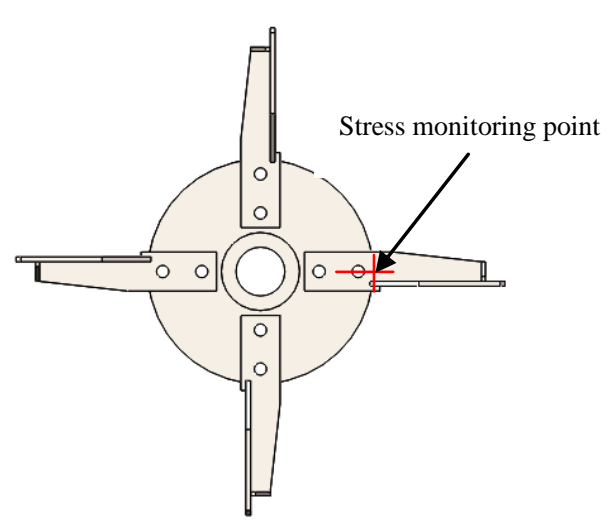

Figure 2. The measuring point distribution diagram of the impeller's strain

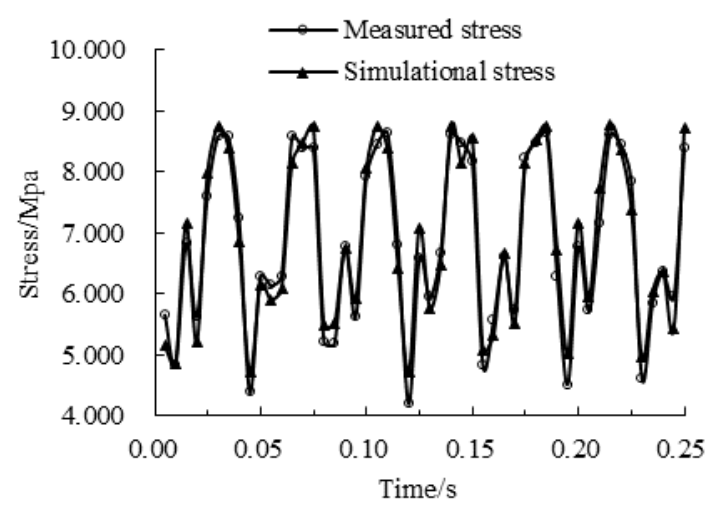

Figure 3. Comparisons between the measured and simulated stress of the impeller under the unloaded condition

\subsubsection{Strain Test of the Throwing Impeller}

In order to verify the correctness of the Finite Element calculation results, the DH5905 wireless strain tester developed by Tung Wah Testing Technologies Co., Ltd. is used to test the strain of the impeller. Considering that the test system would interfere with the throwing performance of materials under the loaded condition, the impeller's stress is measured only for the unloaded condition. The test flow chart is shown in Figure 4.

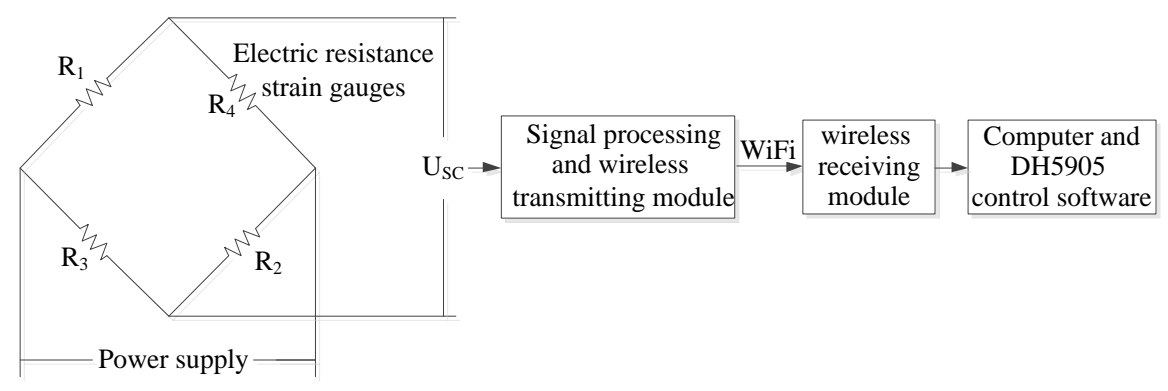

Figure 4. Strain test block diagram by using Dh5905 wireless strain gauge

An AC full-bridge circuit of Wheatstone bridge is formed to measure the impeller's strain. The strain gauges are arranged on the impeller rotating at a high speed of 1500r/min (Figure 5). Because the impeller's strain signals are not convenient for the wire transmission in the closed shell, the DH5905 wireless strain gauge test system is adopted. The entire 
test system, including four strain gauges, one power module, one signal conditioning and wireless transmitting module, one wireless receiving module, and DH5905 signal processing software, is shown in Figure 4. The strain of the impeller under the unloaded condition is measured by the strain gauge. Then, this weak strain signal is amplified and transmitted to the wireless receiving module by the Wheatstone Bridge and signal conditioning and wireless transmitting module. After passing through the $\mathrm{WiFi}$ wireless receiving module, the amplified real-time strain signal is stored on the computer and displayed dynamically on the interface of the DH5905 signal processing software.

Because the maximum stress point is not suitable for pasting the strain gauge, an extension area of the two bolt hole axis of the stiffener near the maximum stress is chosen as the test point shown in Figure 5. For the accurate comparison, the simulated stress monitoring point is set as the same as this position shown in Figure 2. The maximum principal strain direction of the strain gauge is the same as that of the centrifugal force of the impeller. When pasting the strain gauge, the direction of principal strain extends along the center of the two bolt holes. The strain gauges are affixed to the same position on opposite sides of the stiffener. Then, the full-bridge circuit of Wheatstone bridge is constructed with these four strain gauges illustrated in the left side of Figure 4. Because of its own large weight, the wireless transmitting module will be fixed on the hub of the impeller, which will not only reduce the centrifugal force but also reduce the impact on the strain value of the test position. The real connection diagram of strain gauges on the impeller surface and wireless transmitter module are shown in Figure 5. The wireless signal receiving module will be connected to the computer.

After all test instruments are connected, the power is turned on. The throwing impeller's speed is adjusted to $1500 \mathrm{r} / \mathrm{min}$. The stress data acquisition frequency range is set to $200 \mathrm{~Hz}$. The measurement option selects the stress measurement. The measuring range is set to the minimum range. The data must be reset to zero to calibrate the testing strain data before collecting the new strain data.

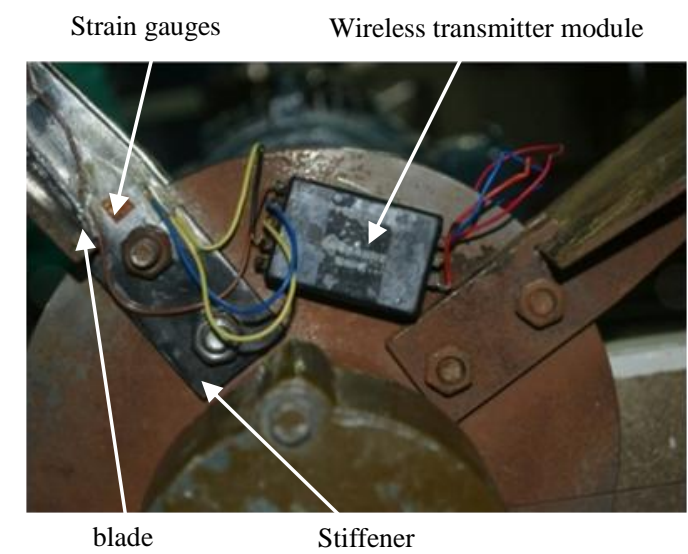

Figure 5. The connection diagram of strain gauges and wireless transmitting module

\subsubsection{Comparison Between Measured Data and Simulated Data}

The comparison chart of the simulated value and the measured value is shown in Figure 3 when the impeller is rotating at $1500 \mathrm{r} / \mathrm{min}$ without load. It can be seen that both the simulated stress and measured stress are quasi-periodically fluctuant with the same period and both waveforms are basically consistent. However, there are errors existing between the simulated value and measured value due to the simplification of the numerical calculation, the manufacturing error of the impeller, and the experimental conditions. The simulated maximum stress is slightly larger than the measured maximum stress with a relative error of $1.34 \%$. The minimum simulated stress is greater than the minimum value of the measured stress with an error of $12.51 \%$. This is because the stiffness variation of the impeller installed the wireless transmitting module. The average stress error is $0.77 \%$. Therefore, the simulation results are basically credible.

\subsubsection{Stress Calculation and Analysis of the Working Throwing Impeller under the Loading Condition}

Based on the numerical calculation results under the unloaded condition, the numerical calculation of the gas-solid twophase unsteady flow in the impeller blower under the loading condition is carried out by using the large-eddy simulation and the dense-discrete phase model. The pressure distribution of material and air flow to the impeller is obtained [18]. Similarly, the Fluent and Workbench coupling platform in the Workbench is used to exert the airflow-material two-phase flow field pressure on the coupling surface of the impeller in the numerical calculation. The rest of the numerical calculations are the 
same as the unloaded condition mentioned above.

In the same way, the equivalent stress of the impeller under the loading condition is calculated by using the finite element method as shown in Figure 6. It can be seen that the maximum equivalent stress of the impeller under the loading condition also appears at the junction of the blade root and reinforced plate, which is 27.142MPa. Due to the influence of the throwing material, the maximum equivalent stress of the loading impeller is slightly larger than that of the unloaded impeller. The maximum stress of the impeller under the loading condition changes with time, as shown in Figure 7.

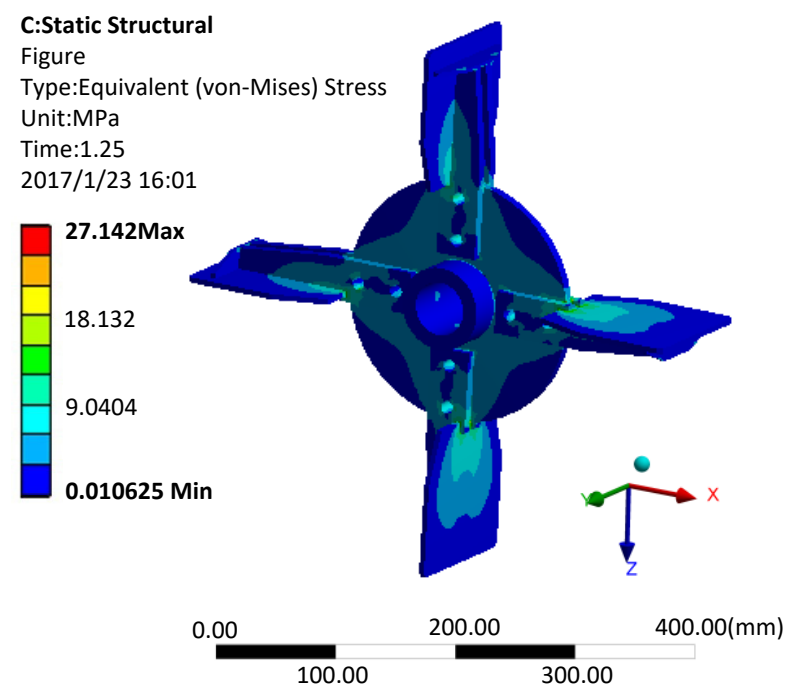

Figure 6. The equivalent stress cloud of the impeller under the loading condition

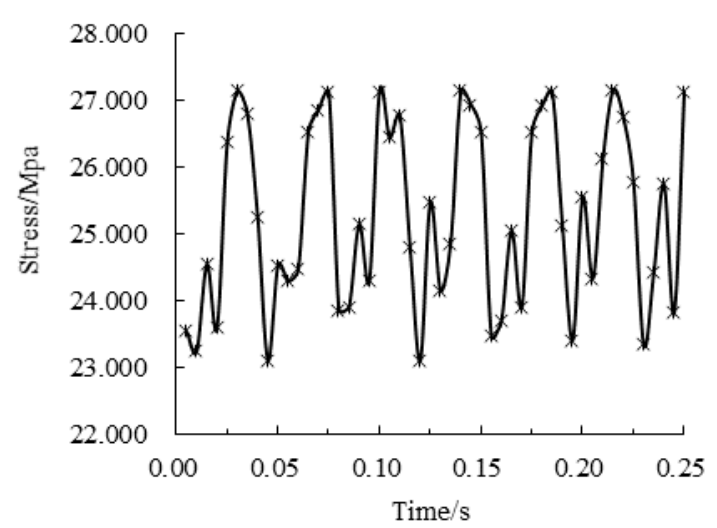

Figure 7. The stress-time variation curve at the maximum stress point of the loading impeller

\section{Fatigue Prediction for the Throwing Impeller}

The material of the throwing impeller is Q235. It can be seen from the fatigue test data of Q235 [16] that its fatigue life obeys the lognormal distribution and the strength limit $S_{b}$ equals $400 \mathrm{MPa}$. According to Equation (1) as well as Equations (16) to (20), the $S-N$ curve parameters can be obtained by fitting the data of the Q235 fatigue test with the least square method [19]. When the reliability is $50 \%, m=8.0677$ and $C=10^{24.54}$. When the reliability is $90 \%, m=8.0677$ and $C=$ $10^{24.29}$. When the reliability is $99 \%, m=8.0677$ and $C=10^{24.23}$.

Considering some factors that affect the fatigue strength of the impeller, takes $K_{S}=1.2, \varepsilon_{S}=0.91, \beta=0.85$, and $\varphi_{S}=0.1$ [15]. Then, the Goodman-type two-parameter nominal stress fatigue life of the impeller can be solved as follows according to Equation (12).

When the reliability is $50 \%$,

$$
\left[\frac{1241.1118 S_{a}}{1.5514(1+r) S_{a}+(1-r)\left(400-0.1 S_{m}\right)}\right]^{8.0677} N=10^{24.54}
$$

When the reliability is $90 \%$,

$$
\left[\frac{1241.1118 S_{a}}{1.5514(1+r) S_{a}+(1-r)\left(400-0.1 S_{m}\right)}\right]^{8.0677} N=10^{24.29}
$$

When the reliability is $99 \%$, 


$$
\left[\frac{1241.1118 S_{a}}{1.5514(1+r) S_{a}+(1-r)\left(400-0.1 S_{m}\right)}\right]^{8.0677} N=10^{24.23}
$$
follows.

According to Equation (13), the Gerber-type two-parameter nominal stress fatigue life of the impeller can be solved as

When the reliability is $50 \%$,

$$
\left\{\frac{\left[(1-r)^{2}\left(400^{2}-0.01 S_{m}^{2}\right)^{2}+4 \times 1.5514^{2} \times 400^{2} \times S_{a}^{2}(1+r)^{2}\right]^{1 / 2}-(1-r)\left(400^{2}-0.01 S_{m}^{2}\right)}{1.5514 S_{a}(1+r)^{2}}\right\}^{8.0677} N=10^{24.54}
$$

When the reliability is $90 \%$,

$$
\left\{\frac{\left[(1-r)^{2}\left(400^{2}-0.01 S_{m}^{2}\right)^{2}+4 \times 1.5514^{2} \times 400^{2} \times S_{a}^{2}(1+r)^{2}\right]^{1 / 2}-(1-r)\left(400^{2}-0.01 S_{m}^{2}\right)}{1.5514 S_{a}(1+r)^{2}}\right\}^{8.0677} \quad N=10^{24.29}
$$

When the reliability is $99 \%$,

$$
\left\{\frac{\left[(1-r)^{2}\left(400^{2}-0.01 S_{m}^{2}\right)^{2}+4 \times 1.5514^{2} \times 400^{2} \times S_{a}^{2}(1+r)^{2}\right]^{1 / 2}-(1-r)\left(400^{2}-0.01 S_{m}^{2}\right)}{1.5514 S_{a}(1+r)^{2}}\right\}^{8.0677} \quad N=10^{24.23}
$$

According to Figure 7, the stress amplitude $S_{a}$, the mean stress $S_{m}$, and the stress ratio $r$ at the impeller's dangerous section can be obtained. When the throwing impeller's reliability $P$ is $50 \%, 90 \%$, and $99 \%$, its fatigue life at $1500 \mathrm{r} / \mathrm{min}$ can be calculated respectively in Table 1 according to Formulas (12), (13), and (15). In order to evaluate the estimation accuracy of the two-parameter nominal stress model, its fatigue life prediction results are compared with those of the conventional $S$ $N$ curve as well as the rating lives of the impeller in Table 1. When the conventional $S-N$ curve is used to estimate the fatigue lives of the impeller, the random cycle load applied on the impeller has to be equivalently converted into a symmetrical cycle load.

Table 1. Comparisons between predicting lives and rating lives of the throwing impeller (h)

\begin{tabular}{|c|c|c|c|}
\hline$P(\%)$ & 50 & 90 & 99 \\
\hline Conventional $S$ - $N$ curve (Gerber) & $1.2780 \times 10^{7}$ & $7.1867 \times 10^{6}$ & $6.2593 \times 10^{6}$ \\
\hline Conventional $S$ - $N$ curve (Goodman) & $7.7963 \times 10^{6}$ & $4.3842 \times 10^{6}$ & $3.8195 \times 10^{6}$ \\
\hline Two-parameter model (Goodman) & $6.1391 \times 10^{6}$ & $3.4522 \times 10^{6}$ & $3.0068 \times 10^{6}$ \\
\hline Two-parameter model (Gerber) & $3.2761 \times 10^{6}$ & $1.8679 \times 10^{6}$ & $1.6046 \times 10^{6}$ \\
\hline Rating life & $3.5 \times 10^{6}$ & $2.0 \times 10^{6}$ & $1.8 \times 10^{6}$ \\
\hline
\end{tabular}

The comparison between the rating lives and the predicted fatigue lives of the two-parameter nominal stress model and the conventional $S-N$ curve showed that the impeller's actual design lives approach the calculation results of the Goodman and Gerber two-parameter nominal stress model. They especially come close to those of the Gerber-type two-parameter nominal stress model and are far below those of the conventional $S-N$ curve. This means the estimating accuracies of the Goodman and Gerber two-parameter nominal stress models are obviously higher than those of the conventional $S$ - $N$ curve.

\section{Conclusions}

Firstly, the relational expression including the fatigue life, average stress, and stress amplitude is established by using the two-parameter nominal stress model, Miner's fatigue cumulative damage model, and lognormal distribution model of fatigue life. Its parameters can be obtained by finite element analysis. Meanwhile, the random cycle load applied on the impeller does not have to be equivalent to a symmetrical cycle load when using the two-parameter nominal stress model to estimate the fatigue life. The precision of the combined method estimating the fatigue life is improved. 
Secondly, the maximum stress value of the finite element calculation is slightly larger than the measured maximum stress of the throwing impeller. The relative error is $1.34 \%$. The average stress error is $0.77 \%$. The simulated and measured stress variation waveforms and periods are basically same. This shows that the throwing impeller's stress simulation results are credible.

Finally, the comparisons between the rating lives and the predicted lives of the two-parameter nominal stress model and the conventional $S-N$ curve show that the impeller's actual rating lives are closer to the calculated lives of the Goodman and Gerber two-parameter nominal stress models than those of the conventional $S$ - $N$ curve. In particular, they are closer to the calculation results of the Gerber-type two-parameter nominal stress model than those of the Goodman-type two-parameter nominal stress model. This shows that the Gerber-type two-parameter nominal stress model is more accurate and suitable to predict the fatigue life of the throwing impeller.

\section{Acknowledgements}

This research is supported by the Inner Mongolia Natural Science Foundation (No. 2018MS05059), the Inner Mongolia Talent Foundation, and the Outstanding Youth Foundation of the Inner Mongolia Agricultural University (No. 2014XYQ-9).

\section{References}

1. Z. P. Zhai, L. Zhou, Z. Y. Yang, Y. Q. Zhao, and S. M. Gan, "Analysis on Vibration Characteristics of Throwing Impeller of Stalk Impeller Blower," Transactions of the Chinese Society of Agricultural Engineering (Transactions of the CSAE), Vol. 31, No. 4, pp. 17-25, 2015 (in Chinese with English abstract)

2. Z. P. Zhai, Y. Q. Zhao, and H. M. Cui, "Modal Analysis and Structure Optimization for the Throwing Impeller of the Stalk Rubbing Machine," International Agricultural Engineering Journal, Vol. 25, No. 4, pp. 72-83, 2016

3. P. S. Chattopadhyay and P. S. Pandey, "Effect of Knife and Operational Parameters on Energy Requirement in Flail Forage Harvesting," Agricultural Engineering Research, Vol. 73, No. 2, pp. 3-12, 1999

4. S. Krzysztof and L. Aleksander, "Two-Stage Motion of Particles in the Discharge Spout of Forage Harvester," Agricultural Engineering, Vol. 124, No. 6, pp. 245-252, 2010

5. A. Lisowski, K. Świątek, J. Klonowski, M. Sypuła, and J. Chlebowski, "Movement of Chopped Material in the Discharge Spout of Forage Harvester with a Flywheel Chopping Unit: Measurements using Maize and Numerical Simulation," Biosystems Engineering, Vol. 111, No. 4, pp. 381-391, 2012

6. Z. P. Zhai, Z. Y. Yang, B. Gao, and J. X. Li, "Simulation of Solid-Gas Two-Phase Flow in an Impeller Blower based on Mixture Model," Transactions of the Chinese Society of Agricultural Engineering (Transactions of the CSAE), Vol. 29, No. 22, pp. 50-58, 2013 (in Chinese with English abstract)

7. S. Dileep, S. E. Muthu, P. Udayanan, and R. K. Mishra, "Multiaxial Fatigue Damage Prediction and Life Estimation of a Centrifugal Impeller for a Turboshaft Engine," Journal of Failure Analysis \& Prevention, Vol. 16, No. 6, pp. 883-891, 2015

8. E. M. Shanmugam, R. V. Prakash, and S. Ammaiappan, "Probabilistic Fatigue Life Assessment of a Titanium Alloy Impeller for Turbo Shaft Engine Application," in Proceedings of ASME 2015 Gas Turbine India Conference, 2015

9. L. Xu, H. J. Cao, H. L. Liu, and Y. B. Zhang, "Assessment of Fatigue Life of Remanufactured Impeller based on FEA," Frontiers of Mechanical Engineering, No. 3, pp. 219-226, 2016

10. Q. Liao, H. Z. Huang, S. P. Zhu, and D. Ling, "Turbine Disk Fatigue Life Prediction based on Generalized $\sigma-N$ Surface," Journal of University of Electronic Science and Technology of China, Vol. 42, No. 2, pp. 316-320, 2013

11. D. G. Pavlou, "The Theory of the S-N Fatigue Damage Envelope: Generalization of Linear, Double-Linear, and Non-Linear Fatigue Damage Models," International Journal of Fatigue, Vol. 110, pp. 204-214, 2018

12. M. Saggar, H. Sallem, and C. Bouraoui, "Fatigue Life Prediction under Variable Loading based on a New Damage Model Devoted for Defective Material," International Journal of Advanced Manufacturing Technology, Vol. 95, No. 1-4, pp. 431-443, 2018

13. M. L. Aggarwal, V. P. Agrawal, and R. A. Khan, "A Stress Approach Model for Predictions of Fatigue Life by Shot Peening of EN45A Spring Steel," International Journal of Fatigue, Vol. 28, No. 12, pp. 1845-1853, 2006

14. S. Ishihara, A. J. Mcevily, M. Sato, K Taniguchi, and T Goshima, "The Effect of Load Ratio on Fatigue Life and Crack Propagation Behavior of an Extruded Magnesium Alloy," International Journal of Fatigue, Vol. 31, No. 11-12, pp. 1788-1794, 2009

15. S. Z. Zhou, K. Q. Zhu, H. C. Wu, and C. Qiao, "Fatigue Life Analysis of the Blender Truck Mixing Impeller based on Workbench," China Petroleum Machinery, No. 2, pp. 36-38, 2012

16. J. L. Fan, X. L. Guo, C. W. Wu, and D. W. Deng, "Fast Evaluation of Fatigue Behavior of Q235 Steel by Infrared Thermography and Energy Approach," Journal of Materials Engineering, Vol. 40, No. 12, pp. 71-76, 2012

17. W. X. Liu, "Mechanical Reliability Design," Tsinghua University Press, Beijing, 1996

18. Z. P. Zhai, L. Zhang, C. Z. Liu, H. N. Li, and H. M. Cui, "Numerical Simulation and Experimental Validation of Radiation Noise from Vibrating Shell of Stalk Impeller Blower," Transactions of the Chinese Society of Agricultural Engineering (Transactions of the CSAE), Vol. 33, No. 16, pp. 72-79, 2017 (in Chinese with English abstract)

19. J. L. Fan, X. L. Guo, C. W. Wu, and Y. G. Zhao, "Research on Fatigue Behavior Evaluation and Fatigue Fracture Mechanisms of Cruciform Welded Joints," Materials Science \& Engineering A, Vol. 528, No. 29-30, pp. 8417-8427, 2011 